\title{
The new idea of scalp electroacupuncture improving upper limb motor function in convalescent phase of ischemic stroke
}

Tong sheng $\mathrm{Su}^{*}$

Shaanxi Hospital of Traditional Chinese Medicine, China

Upper limb (UL) impairment is common in patients of ischemic stroke and recovery of motor function is important for regaining independence in activities of daily living. Researches show that, the death toll of people who are suffering from stroke is more than 200 millions, the annual growth rate of this data reached $8.7 \%$, among which the patient with ischemic stroke takes $60-70 \%$, and who suffers from UL impairment reaches about $70 \%$. At present, the morbidity of ischemic stroke in China ranks first among the world. Aging is a risk for ischemic stroke, especially over the age of 50 years old, which will increase morbidity rate of UL motor function impairment. UL dysfunction makes patients despaired and fail to attend social activity normally, decreasing the quality of their lives. Participation in social activity is very important for people's wellbeing, or they will get a series of diseases, like disability, depressive disorder, etc.

In China, there are many methods for the treatment of upper limb motor dysfunction following scalp acupuncture treatment, such as Jiao scalp acupuncture, Fang scalp acupuncture, Tang scalp acupuncture, etc. Afterwards, China Association for Acupuncture and Moxibustion also developed an international standardization plan for scalp acupuncture points and implemented by the International Health Organization. However, most of these methods use the meridian theory of traditional Chinese medicine or cortical anatomy as the basis of selecting acupoints. In fact, these methods are not specific. In recent years, in the international community, in order to improve upper limb motor dysfunction after stroke, more non-invasive and safe scalp stimulation methods have been used, such as transcranial magnetic stimulation, which require different strengths for different patients' brain function status. Adjust the frequency, stimulation site, and coil direction to achieve good treatment results. At present, the transcranial magnetic stimulation technology has been widely used, and the domestic transcranial magnetic stimulation technology has reached the world advanced level, but this treatment is expensive and other problems. Therefore, we envisage combining traditional acupuncture with transcranial magnetic stimulation to investigate whether the application of stimulation parameters of transcranial magnetic stimulation to the scalp electroacupuncture is equally effective in patients with post-stroke upper limb motor dysfunction.

In healthy individuals, the balance of excitability between the two cerebral hemispheres is symmetric. After stroke, the excitability is asymmetric, with the healthy side of the cerebral hemisphere appears pathological excessive active, the affected side appears pathological inhibition. At present, there is a lot of researches on repetitive transcranial magnetic stimulation (rTMS) to promote recovery motor function of stroke patients, using low frequency electroacupuncture stimulation on the contralateral motor cortex, we found this method can reduce the excitability of the contralateral motor cortex. Better clinical outcomes for the affected hand and arm are observed when asymmetry of corticomotor excitability is reduced.

According to the same principle, we will use the scalp electroacupuncture to stimulate the patient's contralateral cerebral cortex motor area, the stimulation parameters of the repetitive transcranial magnetic stimulation used in electric acupuncture the stimulation parameters of the repetitive transcranial magnetic stimulation used in electric acupuncture, in order to achieve similar therapeutic effect.

At present, we are conducting related clinical trials and will use data analysis to prove the effectiveness of scalp electroacpuncture for the improvement of UL motor function after stroke.
Copyright: (C2018 Su TS. This is an open-access article distributed under the terms of the Creative Commons Attribution License, which permits unrestricted use, distribution, and reproduction in any medium, provided the original author and source are credited.
${ }^{*}$ Correspondence to: Tong sheng Su, Shaanxi Hospital of Traditional Chinese Medicine, Xi'an, Shaanxi, 710003, China, Tel: 008615929562568, E-mail: chinasuts@126.com

Received: May 05, 2018; Accepted: May 21, 2018; Published: May 26, 2018 